\title{
Electronic Conductivity in Open Cylindrical Two-Barrier Symmetric Resonance Tunnel Structure
}

\author{
M.V. Tkach, O.M. Makhanets, Ju.O. Seti, M.M. Dovganiuk \\ AND O.M. VOITSEKHIVSKA \\ Chernivtsi National University, Chernivtsi, Kotsiybynsky str. 2, 58012, Ukraine
}

(Received February 2, 2010; in final form April 6, 2010)

\begin{abstract}
The theory of resonance energies and widths of electron quasi-stationary states and electronic conductivity in open cylindrical two-barrier symmetric resonance tunnel structure is developed. The complete Schrodinger equation is solved within the model of effective masses for rectangular potential wells and barriers. Interaction between electrons and electromagnetic field was taken into account using the approximation of the small signal. The calculations of spectral parameters are performed for $\operatorname{In}_{0.53} \mathrm{Ga}_{0.47} \mathrm{As} / \mathrm{In}_{0.52} \mathrm{Al}_{0.48} \mathrm{As}$ resonance tunnel structure. The dependences of conductivity on the energy of mono-energy electrons beam falling at the system and electromagnetic field energy absorbed or emitted by the system are obtained and analyzed. The relation between experimentally measured parameters of conductivity and resonance widths of electron quasi-stationary states in open resonance tunnel structure is established.
\end{abstract}

PACS numbers: 73.21.Hb, 73.21.La, 73.40.Gk

\section{Introduction}

The rapid development of nano-technologies brings to the creation of new devices with unique physical characteristics. Their elements are different nanostructures: quantum dots, wires and wells, in general and resonance tunnel structures (RTS), in particular. Growing interest to the latter is caused by the fact that after creation of the first quantum cascade (QC) lasers by J. Faist and F. Capasso [1, 2], evident success is achieved in the design of active nano-structures [3,4], minimizing guided currents and reaching the maximal strength of electromagnetic radiation in the demanded range of frequencies (especially, in actual Terahertz ones).

The well known problem of modern QC lasers is the non coherent regime of their action (respectively the electrons), at which the interaction of electrons and RTS dissipative subsystems, such as phonons or impurities, strongly changes the phase of electronic current propagating through the system. Consequently, the effectiveness of QC laser emission becomes small. One of the way of improving it, is to use the open RTS, where the life time of electron quasi-stationary states (QSS) is smaller than the duration of dissipative processes in the system. Such open RTS (mainly quasi-two-dimensional) are investigated for more than ten years using different theoretical models [5-9]. However, the consistent theory of their response is still far from the completion.

The other problem is that for the calculation of electronic current in open RTS (or dynamic conductivity) it is necessary to solve the complete Schrodinger equation with Hamiltonian, taking into account the interac- tion between electrons and electromagnetic field. The mathematical complication is that in the majority of theoretical papers [10-17] simplified models of nano-systems (mainly the plane ones) are used. These models are based at the $\delta$-barrier approximation of RTS rectangular potential barriers. The evident disadvantage of $\delta$-barrier model is the total neglect of the different electron effective masses in nano-system wells and barriers. It is shown in [18], that it essentially influence at the spectral parameters (resonance energies and particularly widths) of electron QSS and through the electronic current conductivity - at the strength of electromagnetic radiation and its frequency.

It is well known that the main parameters of nano-devices, as a rule, can be improved decreasing the spatial dimension of their elements. Thus, during the last years the widening of experimental interest is observed for the research of quantum wires, dots and combined nano-systems $[19,20]$. As far as we know, there are not a lot of papers concerning the theory of quasi-particles spectra (electrons, holes, excitons, etc) and furthermore, the theory of electronic conductivity in open RTS is produced using quantum wires and dots, while the closed RTS are intensively studied [21].

In this paper the theory of spectral parameters is developed (resonance energies (RE) and resonance widths (RW) of electron QSS). We used rather realistic model that consider different electron effective masses in different parts of open cylindrical quantum wire with one quantum well and two rectangular potential barriers. The dynamic conductivity of RTS is calculated within the approximation of small field. 
Besides, it is proven that the conductivity calculated in the framework of the model, as function of injected electrons energy and electromagnetic field energy allows studying not only the field characteristics (radiation or absorption power, frequency, range of maximal emission, etc) but to solve the opposite problem - obtain the RE and RW of electron QSS using the experimentally measured parameters of conductivity.

\section{Conductivity of mono-energy electron beam of cylindrical two-barrier RTS}

Open cylindrical two-barrier RTS with geometrical parameters is shown in Fig. 1a. We assume that the current of uncoupling electrons with concentration $n$ falls at the RTS from the left side propagating in the direction parallel to the axial axis. The electron effective masses and potential energies in different RTS parts in cylindrical coordinate system $(\rho, \varphi, z)$ are the following:

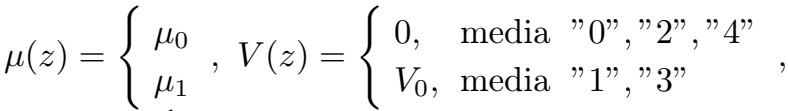

$$
\begin{aligned}
& v(\rho)=\left\{\begin{array}{ll}
0, & \rho<\rho_{0} \\
\infty, & \rho>\rho_{0}
\end{array}\right. \text {. }
\end{aligned}
$$
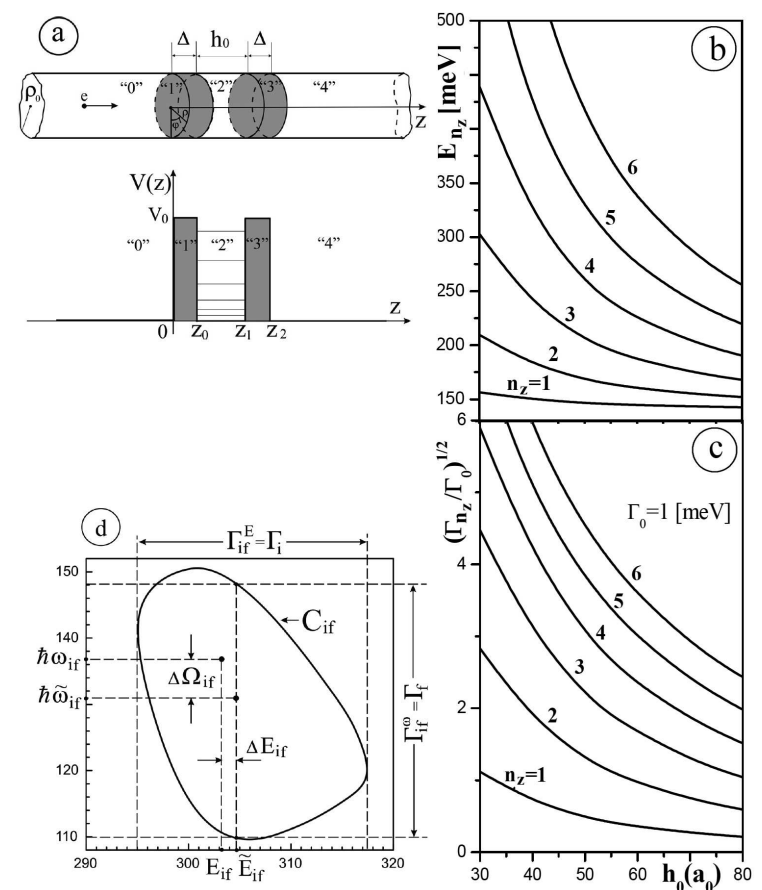

Fig. 1. (a) Geometrical and potential energy schemes of open cylindrical two-barrier RTS, (b) dependences of resonance energy $E_{n_{z}}$ and (c) resonance width $\left(\Gamma_{n_{z}} / \Gamma_{0}\right)^{1 / 2}$ on quantum dot height, (d) dependence of resonance energy $E_{n_{2}}$ on $h_{0}$ and conductivity contour $C_{i f}$.

The electronic conductivity in RTS is fixed by the density of electronic current $j$ flowing through the nano-system, in its turn, defined by the wave function of electron interacting with varying in time electromagnetic field: $\varepsilon(t)=\varepsilon\left(\mathrm{e}^{\mathrm{i} \omega t}+\mathrm{e}^{-\mathrm{i} \omega t}\right)$, with frequency $\omega$ and amplitude of electric field strength $\varepsilon$. The wave function $\Psi(r, t)$ satisfies the complete Schrodinger equation:

$$
\mathrm{i} \hbar \frac{\partial \Psi(r, t)}{\partial t}=(\hat{H}(r)+\hat{H}(z, t)) \Psi(r, t),
$$

where the basic Hamiltonian of electron (in the stationary case) is given by:

$$
\begin{aligned}
& \widehat{H}(\rho, \varphi, z)=-\frac{\hbar^{2}}{2}\left[\frac{\partial}{\partial z} \frac{1}{\mu(z)} \frac{\partial}{\partial z}\right. \\
& \left.\quad+\frac{1}{\mu(z) \rho}\left(\frac{\partial}{\partial \rho} \rho \frac{\partial}{\partial \rho}+\frac{1}{\rho} \frac{\partial^{2}}{\partial \varphi^{2}}\right)\right]+V(z)+v(\rho) .
\end{aligned}
$$

It contains the first term - kinetic energy operator, the second one - potential energy of electron in axial direction, and the third term - infinite potential confining the electron exit from quantum wire in radial direction.

$$
\begin{aligned}
\widehat{H} & (z, t)=U(z)\left(\mathrm{e}^{\mathrm{i} \omega t}+\mathrm{e}^{-\mathrm{i} \omega t}\right) \\
& =-e \varepsilon\left[z\left(\Theta(z)-\Theta\left(z-z_{2}\right)\right)+z_{2} \Theta\left(z-z_{2}\right)\right] \\
& \times\left(\mathrm{e}^{\mathrm{i} \omega t}+\mathrm{e}^{-\mathrm{i} \omega t}\right)
\end{aligned}
$$

is the Hamiltonian of interaction between the electron and varying in time electromagnetic field.

In order to solve the complete Schrodinger equation, it is necessary to solve the stationary one:

$$
\hat{H}(r) \Psi(r)=E \Psi(r),
$$

written for the electron with effective mass $\mu(z)$, that moves in cylindrical quantum wire as in closed system in radial direction, and as in open system - in axial one. The wave function $\Psi(r)$ is given by:

$$
\Psi(\rho, \varphi, z)=\Psi_{n_{\rho} m}(\rho, \varphi) F(z),
$$

and, therefore, the variables in Eq. (5) are separated. The energy spectrum

$$
E_{n_{\rho} m}=\frac{\hbar^{2} x_{n_{\rho} m}^{2}}{2 \mu_{0} \rho_{0}^{2}},
$$

and normalized wave functions [21]:

$$
\begin{aligned}
& \Psi_{n_{\rho} m}(\rho, \varphi)=\left(\pi \rho_{0}^{2}\left|J_{m-1}\left(x_{n_{\rho} m}\right) J_{m+1}\left(x_{n_{\rho} m}\right)\right|\right)^{-1 / 2} \\
& \quad \times J_{m}\left(X_{n_{\rho} m} \rho_{0}^{-1} \rho\right) \mathrm{e}^{\mathrm{i} m \varphi}
\end{aligned}
$$

describe the confined movement of electron (with mass $\left.\mu_{0}\right)$ in the plane perpendicular to the axial axis. Here $J_{m}$ is the Bessel function of the first order, $X_{n_{\rho} m}$ are the roots of Bessel function, $m=0 ; \pm 1 ; \pm 2 \ldots$ is magnetic quantum number, $n_{\rho}=1,2,3 \ldots$ - radial quantum number fixing the number of Bessel function at certain $m$.

Now, taking into account (5), (6), (8), the wave functions $F_{n_{\rho} m}(z)$ evidently satisfy the equation:

$$
\begin{aligned}
& {\left[-\frac{\hbar^{2}}{2} \frac{\partial}{\partial z} \frac{1}{\mu(z)} \frac{\partial}{\partial z}+V(z)+E_{n_{\rho} m}\right] F_{n_{\rho} m}(z)} \\
& \quad=E F_{n_{\rho} m}(z),
\end{aligned}
$$

with open fitting conditions. This equation is equally 
solved for any $\mid n_{\rho} m>$ states. Further, we are going to study in details the conductivity of RTS of rather small radius, where the only state $\left(n_{\rho}=1, m=0\right)$ is realized in the actual sub-barrier region. We put $F_{10}(z)=F_{0}(z)$, $E_{0}=\frac{\hbar^{2} x_{10}^{2}}{2 \mu_{0} \rho_{0}^{2}}$ and obtain from Eq. (2) the equation for $F(z, t)$ function:

$$
\begin{aligned}
& \mathrm{i} \hbar \frac{\partial F(z, t)}{\partial t}=\left[-E_{0}-\frac{\hbar^{2}}{2} \frac{\partial}{\partial z} \frac{1}{\mu(z)} \frac{\partial}{\partial z}+V(z)\right. \\
& \quad+\widehat{H}(z, t)] F(z, t) .
\end{aligned}
$$

This equation can be solved within the approximation of small signal [5-10], assuming that the amplitude of the electric field $\varepsilon$ is small. Thus, its general solution is written as:

$$
F(z, t)=F_{0}(z) \mathrm{e}^{-\mathrm{i} \omega_{0} t}+F_{1}(z, t),
$$

where $\omega_{0}=\hbar^{-1} E, F_{0}(z)$ function is the solution of stationary Schrodinger equation (9) and the correction of the first order in one-mode approximation is found as:

$$
F_{1}(z, t)=F_{+1}(z) \mathrm{e}^{-\mathrm{i}\left(\omega_{0}+\omega\right) t}+F_{-1}(z) \mathrm{e}^{-\mathrm{i}\left(\omega_{0}-\omega\right) t} .
$$

Preserving the magnitudes of the first smallness order and taking into account formulae (10), and (11), the equation for the both parts of $F_{1}(z, t)$ functions is obtained:

$$
\begin{aligned}
& {\left[-\frac{\hbar^{2}}{2} \frac{\partial}{\partial z} \frac{1}{\mu(z)} \frac{\partial}{\partial z}+V(z)+E_{0}-\hbar\left(\omega_{0} \pm \omega\right)\right] F_{ \pm 1}(z)} \\
& \quad=U(z) F_{0}(z)
\end{aligned}
$$

The stationary case (9) with open boundaries is solved exactly and $F_{0}(z)$ wave function is:

$$
\begin{aligned}
& F_{0}(z)=F_{0}^{(0)}(z) \Theta(-z)+F_{0}^{(4)} \Theta\left(z-z_{2}\right) \\
& \quad+\sum_{p=0}^{2} F_{0}^{(p+1)}\left[\Theta\left(z-z_{p-1}\right)-\Theta\left(z-z_{p}\right)\right] \\
& =\left(A_{0} \mathrm{e}^{\mathrm{i} k_{0} z}+B_{0} \mathrm{e}^{-\mathrm{i} k_{0} z}\right) \Theta(-z) \\
& \quad+A_{4} \mathrm{e}^{\mathrm{i} k_{0}\left(z-z_{2}\right)} \Theta\left(z-z_{2}\right) \\
& \quad+\sum_{p=0}^{2}\left(A_{p+1} \mathrm{e}^{\mathrm{i} k_{p+1} z}+B_{p+1} \mathrm{e}^{-\mathrm{i} k_{p+1} z}\right) \\
& \quad \times\left[\Theta\left(z-z_{p-1}\right)-\Theta\left(z-z_{p}\right)\right]
\end{aligned}
$$

where $z_{-1}=0, k_{0}(E)=k_{2}(E)=\sqrt{\frac{2 \mu_{0}}{\hbar^{2}} E-\frac{X_{n_{\rho} m}^{2}}{\rho_{0}^{2}}}$, $k_{1}(E)=k_{3}(E)=\sqrt{\frac{2 \mu_{1}}{\hbar^{2}}\left(E-U_{0}\right)-\frac{X_{n_{\rho} m}^{2}}{\rho_{0}^{2}}}$.

All unknown coefficients $\left(A_{p}\right.$ and $\left.B_{p}(p=0,1,2,3,4)\right)$ are fixed by the conditions for wave function and its density of current continuity at all nano-system interfaces:

$$
\begin{aligned}
& F_{0}^{(p)}\left(z_{p-1}\right)=F_{0}^{(p+1)}\left(z_{p-1}\right), \\
& \left.\frac{1}{\mu_{p}} \frac{\mathrm{d} F_{0}^{(p)}}{\mathrm{d} z}\right|_{z=z_{p-1}}=\left.\frac{\mathrm{d} F_{0}^{(p+1)}}{\mu_{p+1} \mathrm{~d} z}\right|_{z=z_{p-1}},
\end{aligned}
$$

$$
p=0,1,2,3 .
$$

The solutions of inhomogeneous Eqs. (13) are the superpositions of functions:

$$
F_{ \pm 1}(z)=F_{ \pm}(z)+\Phi_{ \pm}(z)
$$

where $F_{ \pm}(z)$ are the solutions of homogeneous Eqs. (13).

$$
\begin{aligned}
& F_{ \pm}(z)=F_{ \pm}^{(0)}(z) \Theta(-z)+F_{ \pm}^{(4)}(z) \Theta\left(z-z_{2}\right) \\
& +\sum_{p=0}^{2} F_{ \pm}^{(p+1)}\left[\Theta\left(z-z_{p-1}\right)-\Theta\left(z-z_{p}\right)\right] \\
& =B_{0}^{ \pm} \mathrm{e}^{-\mathrm{i} k_{0}^{ \pm} z} \Theta(-z)+A_{4}^{ \pm} \mathrm{e}^{\mathrm{i} k_{0}^{ \pm}\left(z-z_{2}\right)} \Theta\left(z-z_{2}\right) \\
& +\sum_{p=0}^{2}\left(A_{p+1}^{ \pm} \mathrm{e}^{\mathrm{i} k_{p+1}^{ \pm}}+B_{p+1}^{ \pm} \mathrm{e}^{-\mathrm{i} k_{p+1}^{ \pm}}\right) \\
& \times\left[\Theta\left(z-z_{p-1}\right)-\Theta\left(z-z_{p}\right)\right],
\end{aligned}
$$

where

$$
\begin{aligned}
& k_{0}^{ \pm}(E)=k_{2}^{ \pm}(E)=k_{0}(E \pm \hbar \omega), \\
& k_{1}^{ \pm}(E)=k_{3}^{ \pm}(E)=k_{1}(E \pm \hbar \omega),
\end{aligned}
$$

and functions

$$
\begin{aligned}
& \Phi_{ \pm}(z)=\sum_{p=0}^{2}\left(\frac{e \varepsilon}{\mu_{p} \omega^{2}} \frac{\mathrm{d} F_{0}^{(p+1)}}{\mathrm{d} z} \mp \frac{e \varepsilon z}{\hbar \omega} F_{0}^{(p+1)}(z)\right) \\
& \times\left[\Theta\left(z-z_{p-1}\right)-\Theta\left(z-z_{p}\right)\right] \\
& \mp \frac{e \varepsilon z_{2}}{\hbar \omega} F_{0}^{(4)} \Theta\left(z-z_{2}\right)
\end{aligned}
$$

are the exact partial solutions of Eqs. (13). Their general solutions can be written as

$$
\begin{aligned}
& F_{ \pm 1}(z)=F_{ \pm 1}^{(0)}(z) \Theta(-z)+F_{ \pm 1}^{(4)} \Theta\left(z-z_{2}\right) \\
& \quad+\sum_{p=0}^{2} F_{ \pm 1}^{(p+1)}(z)\left[\Theta\left(z-z_{p-1}\right)-\Theta\left(z-z_{p}\right)\right] .
\end{aligned}
$$

The conditions for these wave functions and respective currents continuity at all nano-systems interfaces are analogous to Eqs. (15). They are equivalent to the system of eight linear inhomogeneous equations, determining all eight unknown coefficients $\left(A_{p}^{ \pm}, B_{p}^{ \pm}(p=0,1,2,3,4)\right)$, $F_{ \pm 1}(z)$ and $F(z, t)$ wave functions. According to the quantum mechanics, the density of current in point $z$ at moment of time $t$ of uncoupling electrons with concentration $n$ is given by the formula:

$$
\begin{aligned}
& j(z, t)=\frac{e \hbar n}{2 \mu(z)} \\
& \quad \times\left(F(z, t) \frac{\partial}{\partial z} F^{*}(z, t)-F^{*}(z, t) \frac{\partial}{\partial z} F(z, t)\right) .
\end{aligned}
$$

In the framework of quasi-static approximation, taking into account that the lengths of electromagnetic waves interacting with electron are much bigger than the sizes of RTS one can calculate the energy $W$ received (or given) by electron from the field during the period of time 


$$
\begin{aligned}
& T=2 \pi / \omega: \\
& W=\int_{0}^{\mathrm{T}} \mathrm{d} t \int_{0}^{z_{2}} j(z, t) \varepsilon(z, t) \mathrm{d} z=2 T d \sigma_{g} \varepsilon^{2},
\end{aligned}
$$

where $\sigma_{g}=\Re \sigma$ and $\sigma$ are complex conductivities of nano-system.

The same energy can be obtained as a sum of energies of electromagnetic waves coming out of the both sides of quantum wire:

$$
\begin{aligned}
W & =\frac{\hbar \omega T}{e}\left\{\left[j\left(E+\hbar \omega, z=z_{2}\right)-j\left(E-\hbar \omega, z=z_{2}\right)\right]\right. \\
& -[j(E+\hbar \omega, z=0)-j(E-\hbar \omega, z=0)]\} .
\end{aligned}
$$

Combining the Eqs. (19) and (20) and calculating the densities of currents, the analytical expression for the real part of conductivity is obtained:

$$
\begin{aligned}
& \sigma_{g}(E, \omega)=\frac{\hbar^{2} \omega n}{2 z_{2} \mu_{0} \varepsilon^{2}}\left[k_{0}^{+}\left(\left|B_{0}^{+}\right|^{2}+\left|A_{4}^{+}\right|^{2}\right)\right. \\
& \left.-k_{0}^{-}\left(\left|B_{0}^{-}\right|^{2}+\left|A_{4}^{-}\right|^{2}\right)\right],
\end{aligned}
$$

which has the clear physical meaning. The first two terms describe the contributions into nano-system conductivity of electron wave currents from RTS left side $B_{0}^{+}$and right side $A_{4}^{+}$with radiation, the rest two terms describe the contributions of currents from left side $B_{0}^{-}$and from right side $A_{4}^{-}$with absorbing of electromagnetic waves with frequency $\omega$. It is to be mentioned that, in the proposed approach, coefficients $B_{0}^{ \pm}$and $A_{4}^{ \pm}$are proportional to $\varepsilon^{2}$, therefore $\sigma_{g}$ magnitude is independent of the fields strength.

The further calculation and analysis of spectral parameters (RE and RW) of electron QSS and its conductivity $\sigma_{g}$ is performed for $\mathrm{In}_{0.53} \mathrm{Ga}_{0.47} \mathrm{As} / \mathrm{In}_{0.52} \mathrm{Al}_{0.48} \mathrm{As} \mathrm{RTS}$, often researched experimentally $[3,4]$.

\section{Discussion of results}

The main properties of electronic conductivity $\sigma_{g}$, as function of electrons energy $E$ and electromagnetic field frequency $\omega$ emitted or absorbed are completely determined by RE and RW of electron QSS, that in turn, depend on geometric size and energy parameters of nano-system. The calculation of $\sigma_{g}$ magnitude is performed for $\mathrm{In}_{0.53} \mathrm{Ga}_{0.47} \mathrm{As} / \mathrm{In}_{0.52} \mathrm{Al}_{0.48} \mathrm{As}$ cylindrical open symmetric two-barrier RTS with material parameters: electron effective masses $\mu_{0}=0.046 m_{0}, \mu_{1}=0.089 m_{0}\left(m_{0}\right.$ is pure electron mass); lattice constants $a_{0} \approx a_{1}=5.87 \mathrm{~A}^{\circ}$, height of the potential barrier $V_{0}=516 \mathrm{meV}$.

The RE $E_{n_{z}}$ and RW $\Gamma_{n_{z}}$ of $n_{z}$-th QSS are defined by the location of the maximum of the peak of penetration coefficient $D(E)$ of electron through the RTS in energy scale and its width in the same scale at the half of the heights, respectively. The typical dependencies of $E_{n_{z}}$ and $\Gamma_{n_{z}}$ on the height $h_{0}$ of cylindrical quantum dot, at $\rho_{0}=10 a_{0}, \Delta=2 a_{1}$, are shown in Figs. $1 \mathrm{~b}$ and c.

From the figures one can see that at the increasing of quantum well size $h_{0}$ the REs are decreasing approximately quadratically and RWs - more rapidly. It is clear because the increasing of quantum dot height $h_{0}$ makes its volume bigger, since all REs become smaller and the "effective height" of potential barrier becomes bigger, thus the RWs are sharply decreasing.

(a)
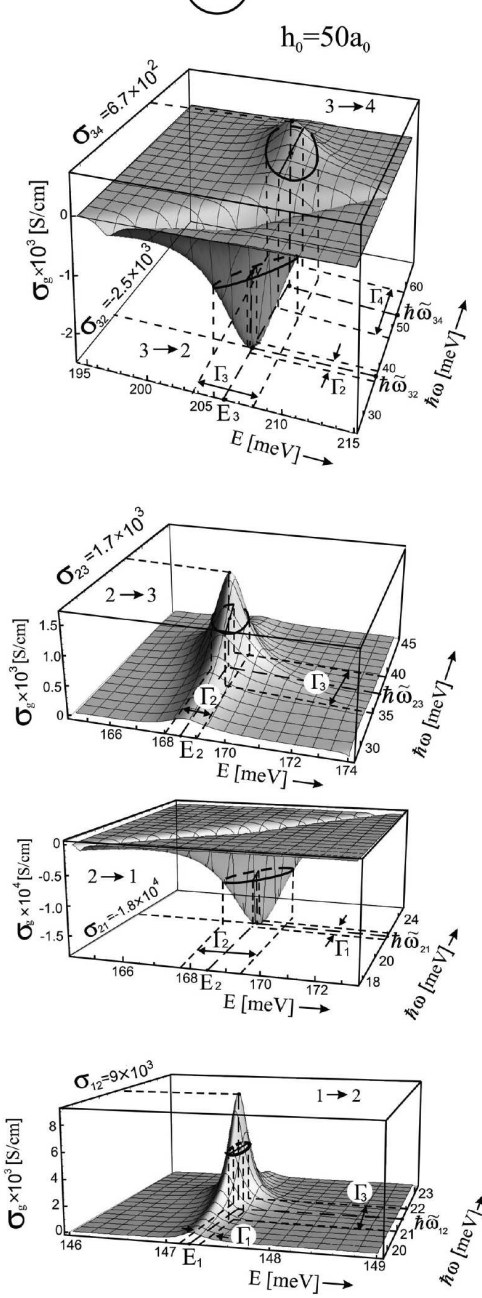

Fig. 2. Dependencies of (a) conductivity $\sigma_{g}$ and (b) its contours $C_{i f}$ on electron beams energy $E$ and electromagnetic field energy $\hbar \omega$ and quantum dot height $h_{0}$ at $\rho_{0}=10 a_{0}, \Delta=2 a_{1}$. Quantum dots height $h_{0}$ is varied for conductivity contours plots.

Figure 2a it is presents the conductivity $\sigma_{g}$ as function of energy $E$ of mono-energetic beam of uncoupled electrons with concentration $n$ and electromagnetic field energy $\hbar \omega$, herein emitted $\left(\sigma_{g}<0\right)$ or absorbed $\left(\sigma_{g}>0\right)$ at $\rho_{0}=10 a_{0}, \Delta=2 a_{1}, h_{0}=50 a_{0}$. The RTS conductivity is formed as a result of quantum transitions of electrons between initial $(i)$ and final $(f)$ states. The transitions from lower to upper QSS $(i<f)$ produce the positive conductivity $\left(\sigma_{g}>0\right)$ and occur with absorption of elec- 
tromagnetic field energy $\hbar \omega$. The transitions from upper into lower QSS $(i>f)$ produce the negative conductivity $\left(\sigma_{g}<0\right)$ and occur with emission of electromagnetic field energy.

It is visible from the Fig. 2a, that function $\sigma_{g}(E, \hbar \omega)$ has extremum $\sigma_{i f}$ caused by electron transitions from $i$-th into $f$-th state $(i \rightarrow f)$, in the points at the plane $(E, \hbar \omega)$, with coordinates over the electron energy $\tilde{E}_{i f}$ and field energy $\hbar \tilde{\omega}_{i f}$ located in the vicinity of RE $E_{i}$ and modulus of the REs difference $\left(\hbar \omega_{i f}=\left|E_{i}-E_{f}\right|\right)$, respectively. The magnitudes $\tilde{E}_{i f}$ and $E_{i}, \hbar \tilde{\omega}_{i f}$ and $\hbar \omega_{i f}$ are a little bit different, thus the concept of electron energy shift $\Delta E_{i f}=\tilde{E}_{i f}-E_{i f}$ and field energy shift $\Delta \Omega_{i f}=$ $\hbar\left(\tilde{\omega}_{i f}-\omega_{i f}\right)$ is introduced. In the figure one can see the main properties of conductivity, depending in the vicinity of which RE $E_{i}$ the electron energy $E$ is located. It is obvious that when $E$ is in the vicinity of $E_{1} \mathrm{RE}$ (the ground QSS), the conductivity is positive in the whole $E$ and $\hbar \omega$ range $\left(\sigma_{g}(E, \omega)>0\right)$. Conductivity maximum $\sigma_{1 f}$ is approached at $\tilde{E}_{1 f}, \hbar \tilde{\omega}_{1 f}$, herein $\sigma_{12} \gg \sigma_{13} \gg \sigma_{14}$. The maximal magnitude of $\sigma_{12}$ is orders bigger than all other $\sigma_{1 f \neq 1}$, consequently, the quantum transition $(1 \rightarrow 2)$ is "allowed" and all other $(i \rightarrow f=2,3 \ldots)$ are "forbidden". When the electron energy $E$ is in the vicinity of RE $E_{i f>2}$, then the extremum magnitudes $\sigma_{i f<i}<0$ and $\sigma_{i f>i}>0$ are approached in the points $\tilde{E}_{i f}, \hbar \tilde{\omega}_{i f}$, herein $\left|\sigma_{i f=i+1}\right| \gg\left|\sigma_{i f=i+2}\right| \gg\left|\sigma_{i f=i+3}\right| \gg \ldots$ Thus, the maximum of $\sigma_{g}(E, \hbar \omega)$ are formed by electron quantum transitions between neighbours QSSs $\sigma_{i i \pm 1}$. The transitions into all other states are formed by the smaller $\sigma_{i f \neq i \pm 1}$, the bigger is $|f-i|$ difference. Therefore, the transitions $(i \rightarrow i \pm 1)$ are "allowed" and all the other are "forbidden".

From physical considerations, it is clear that the properties of conductivity of two-barrier RTS are tightly bound with the properties of REs $E_{i}, E_{f}$ and RWs $\Gamma_{i}, \Gamma_{f}$ of the respective electron QSSs, transitions between which form the magnitudes of $\sigma_{g}$ in the vicinity of the corresponding energies $E$ and frequencies $\omega$. In order to analyze this important relation, it is convenient to introduce the concept of middle contour $C_{i f}$ of conductivity $\sigma_{i f}$, meaning the line of the intersection between $\sigma_{g}(E, \hbar \omega)$ function and the plane parallel to the plane $(E, \hbar \omega)$ being at the half of the height $\sigma_{i f} / 2$, see Fig. 1d.

From Fig. 1d one can see that the projection $\Gamma_{i f}^{\mathrm{E}}$ of middle contour $C_{i f}$ at the energy axis $E$ is almost coinciding with RW $\Gamma_{i}$ of the QSS whose RE $E_{i}$ is nearest to the electron energy. The projection $\Gamma_{i f}^{\omega}$ at $\hbar \omega$ axis of the section created between two cross-points of middle contour $C_{i f}$ and line, parallel to $E$ axis, passing through the point $\left(\tilde{E}_{i f}, \hbar \tilde{\omega}_{i f}\right)$, almost coincide with RW $\Gamma_{f}$ of QSS at which the quantum transition happen. The introduced parameters $\left(\sigma_{i f}, \tilde{E}_{i f}, \hbar \tilde{\omega}_{i f}, \Gamma_{i f}^{\mathrm{E}}, \Gamma_{i f}^{\omega}\right)$ consistently characterize $\sigma_{g}(E, \hbar \omega)$ conductivity, independently of the quantum wire sizes and, at the same time, are the good parameters allowing to obtain REs $E_{i}$ and RWs $\Gamma_{i}$ of electron QSS in open RTS using the experimentally measured $\sigma_{g}$ magnitudes.
Figure $2 \mathrm{~b}$ shows the evolution of middle contours $C_{i f}$ and their parameters at the varying quantum well size $h_{0}$. It is clear that increasing $h_{0}$ for typical transitions does not essentially change the contours shape (topology). The bigger is the electrons energy, the bigger are the sizes of contours $C_{i f}$, herein at $\sigma_{g}>0$ the projection of the contour at $E$ axis: $C_{i f}^{\mathrm{E}} \cong \Gamma_{i f}^{\mathrm{E}} \cong \Gamma_{i}$, projection of contour at $\hbar \omega$ axis: $C_{i f}^{\omega} \cong \Gamma_{i f}^{\omega} \cong \Gamma_{f}$ and at $\sigma_{g}<0$ : projection $C_{i f}^{\mathrm{E}} \cong \Gamma_{i f}^{\mathrm{E}} \cong \Gamma_{i}$, projection $C_{i f}^{\omega} \cong \Gamma_{i f}^{\omega} \cong \Gamma_{i} / 3$.

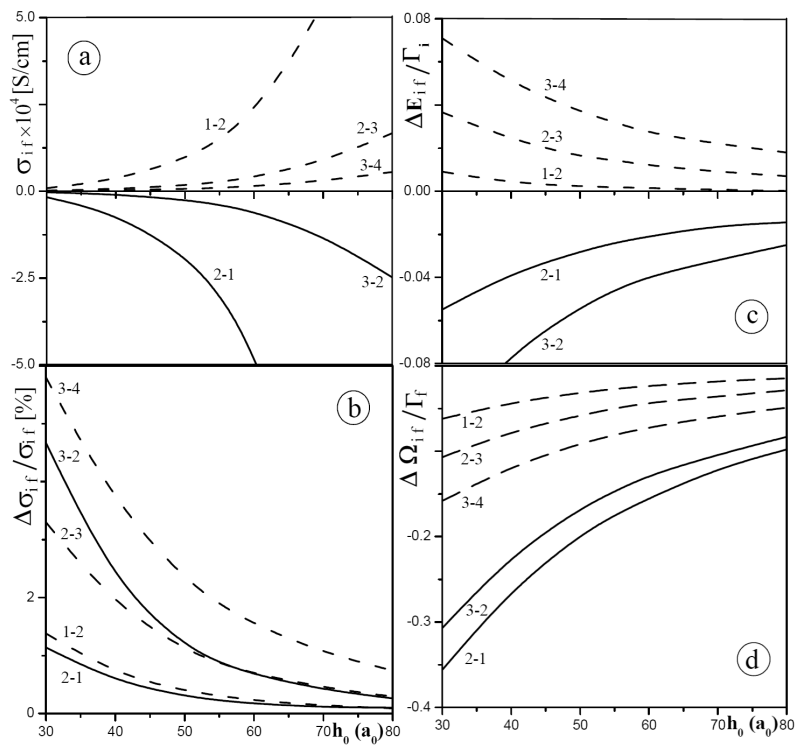

Fig. 3. Dependencies of (a) maximal conductivity $\sigma_{i f}$, (b) relative conductivity shifts $\Delta \sigma_{i f} / \sigma_{i f}$, (c) relative electron energy shift $\Delta E_{i f} / \Gamma_{i}$, (d) relative electromagnetic field energy shift $\Delta \Omega_{i f} / \Gamma_{f}$ on quantum dots height $h_{0}$.

Figure 3 shows the evolution of main parameters of conductivity at the varying quantum well size $h_{0}$ and fixed $\rho_{0}=10 a_{0}, \Delta=2 a_{1}$. It is clear that when $h_{0}$ increases, maximal magnitudes $\sigma_{i f}$ are increasing too, independently of $\sigma_{g}>0$ (transitions $i \rightarrow \mathrm{f}=\mathrm{i}+1$ ) or $\sigma_{g}<$ 0 (transitions $i \rightarrow \mathrm{f}=\mathrm{i}-1$ ). At the fixed RTS sizes, the increasing electrons energy $\mathrm{E}$ brings to the decreasing of maximal conductivity $\sigma_{i f}$ and increasing of relative shifts $\Delta \sigma_{i f}=\sigma_{i f}-\sigma\left(E_{i}, \hbar \omega_{i f}\right) / \sigma_{i f}$. The increases of $h_{0}$ causes the increases of all $\sigma_{i f}$ magnitudes and decreases of all $\operatorname{shifts}\left(\Delta \sigma_{i f} / \sigma_{i f}, \Delta E_{i f} / \Gamma_{i}, \Delta \Omega_{i f} / \Gamma_{f}\right)$.

Finally, we must note that the relative shift over the electron energy $\Delta E_{i f} / \Gamma_{i}$ is positive for the emission processes and negative for the processes of the absorption of electromagnetic waves. The relative shift over the field energy $\Delta \Omega_{i f} / \Gamma_{f}$ is negative, independently of the field energy is emitted or absorbed.

\section{Conclusions}

Within the model of different electron effective masses in different parts of cylindrical RTS with rectangular potential barriers, it is developed the theory of spectral pa- 
rameters (REs and RWs) of electron QSSs. Their relation to the respective parameters of conductivity are established, and calculated as functions of the energy of electrons beam falling at RTS and the electromagnetic field energy (emitted or absorbed).

It is shown that the electron quantum transitions from upper (lower) QSSs into neighbour lower (upper) states formes the negative (positive) dynamic conductivity of the system. The emission (absorption) of electromagnetic waves also occurs, having maximum at the frequency proportional to the difference of QSSs REs between which the transition happens.

Depending on geometric parameters of cylindrical RTS and of electrons beam energies, these systems can be applied as the active elements of the QC laser (at negative conductivity) or nano-sensor (at positive conductivity) in the demanded range of frequencies.

It is proven, that the experimentally measured conductivity of RTS, as function of falling electrons beam energies and electromagnetic field energy, allow defining the conductivity contour whose respective projections directly determine the main spectral parameters (RE and RW) of electron QSS in open RTS.

\section{References}

[1] J. Faist, F. Capasso, D.L. Sivco, C. Sirtori, A.L. Hutchinson, A.Y. Cho, Science 264, 553 (1994).

[2] J. Faist, F. Capasso, C. Sirtori, D.L. Sivco, A.L. Hutchinson, A.Y. Cho, Appl. Phys. Lett. 66, 538 (1995).

[3] C. Gmachl, F. Capasso, D.L. Sivco, A.Y. Cho, Rep. Prog. Phys. 64, 1533 (2001).
[4] A.K.M. Newaz, W. Song, E.E. Mendez, Y. Lin, J. Nitta, Phys. Rev. B 71, 195303 (2005).

[5] H.C. Liu, Appl. Phys. Lett. 52, 453 (1988).

[6] H.C. Liu, Phys. Rev. B 43, 12538 (1991).

[7] R.K. Mains, G.I. Haddad, J. Appl. Phys. 64, 3564 (1988).

[8] R.K. Mains, G.I. Haddad, J. Appl. Phys. 64, 5041 (1988).

[9] V.F. Elesin, Yu. Kopaev, Sol. Stat. Comm. 96, 897 (1995).

[10] E.I. Golant, A.B. Pashkovskii, JETP Lett. 63, 590 (1996).

[11] A.B. Pashkovskii, JETP Lett. 82, 210 (2005).

[12] E.A. Gel'vich, E.I. Golant, A.B. Pashkovskii, Tech. Phys. Lett. 32, 191 (2006).

[13] V.F. Elesin, JETP 89, 377 (1999).

[14] V.F. Elesin, I.Yu. Kateev, Semiconductors 42, 571 (2008).

[15] V.F. Elesin, I.Yu. Kateev, M.A. Remnev, Semiconductors 43, 257 (2009).

[16] Wim Vanroose, Phys. Rev. A 64, 062708 (2001).

[17] A.A. Gorbatsevich, M.N. Zhuravlev, V.V. Kapaev, JETP 107, 288 (2008).

[18] N.V. Tkach, Yu.A. Seti, Low Temp. Phys. 35, 556 (2009).

[19] M.T. Bjork, B.J. Ohlsson, T. Sass, A.I. Persson, C. Thelander, M.H. Magnusson, K. Deppert, L.R. Wallenberg, L. Samuelson, Appl. Phys. Lett. 80, 1058 (2002).

[20] J. Tragardh, A.I. Persson, J.B. Wagner, D. Hessman, L. Samuelson, J. Appl. Phys. 101, 123701 (2007).

[21] N.V. Tkach, A.M. Makhanets, Phys. Sol. Stat. 47, 571 (2005). 